\title{
In the Eye of the Storm
}

\author{
EDWARD J. WALsh, ${ }^{\mathrm{a}}$ C. W. FAIRALl, ${ }^{\mathrm{a}}$ AND IVAN POPSTEFANIJA ${ }^{\mathrm{b}}$ \\ ${ }^{\text {a }}$ NOAA/Physical Sciences Laboratory, Boulder, Colorado \\ ${ }^{\mathrm{b}}$ ProSensing Inc., Amherst, Massachusetts
}

(Manuscript received 11 September 2020, in final form 15 March 2021)

\begin{abstract}
The airborne NOAA Wide Swath Radar Altimeter (WSRA) is a 16-GHz digital beamforming radar altimeter that produces a topographic map of the waves as the aircraft advances. The wave topography is transformed by a two-dimensional FFT into directional wave spectra. The WSRA operates unattended on the aircraft and provides continuous real-time reporting of several data products: 1) significant wave height; 2) directional ocean wave spectra; 3) the wave height, wavelength, and direction of propagation of the primary and secondary wave fields; 4) rainfall rate; and 5) sea surface mean square slope (mss). During hurricane flights the data products are transmitted in real-time from the NOAA WP-3D aircraft through a satellite data link to a ground station and on to the National Hurricane Center (NHC) for use by the forecasters for intensity projections and incorporation in hurricane wave models. The WSRA is the only instrument that can quickly provide high-density measurements of the complex wave topography over a large area surrounding the eye of the storm.
\end{abstract}

KEYWORDS: Hurricanes/typhoons; Sea state; Radars/Radar observations; Aircraft observations

\section{Introduction}

Tamizi and Young (2020) provided an overview of previous observations and computations of the tropical cyclone wave field. They developed an extensive database of tropical cyclone wave field observations from sources which included a global database of satellite altimeter observations from 13 satellite missions over 33 years and in situ data from the U.S. National Data Buoy Center (NDBC) buoy network over 37 years. The combined dataset included altimeter data from 2730 tropical cyclones worldwide and in situ data from 353 hurricanes in the North American region. Their analysis of the combined dataset provides an overview of the spatial distributions of significant wave height and wind speed and the characteristics of the tropical cyclone directional wave spectrum.

Despite the extensive dataset, they noted that the relatively small size of tropical cyclones makes it rare for in situ measurement systems to encounter extreme conditions. And when it does happen, failure of in situ systems is common. They concluded that the relatively small observational database of wave conditions under tropical cyclone forcing limits a complete understanding of the wave fields they generate.

The Tamizi and Young (2020) graphical presentations of the wind and wave characteristics in the vicinity of tropical cyclones, normalized to the radius of maximum wind, impressively demonstrate their general variation. But it is critical to know the characteristics of the particular storm bearing down on you. When their other characteristics are the same, a large category 2 hurricane will produce a larger wave field than a

\footnotetext{
D Denotes content that is immediately available upon publication as open access.
}

Corresponding author: Edward J. Walsh, edward.walsh@ noaa.gov small category 3 , and a fast-moving hurricane will produce larger waves than a slow one.

When it comes to individual storms, in situ measurements provide very little information on the overall system. Collins et al. (2018) discuss the data from two deep-sea moorings deployed $780 \mathrm{~km}$ off the coast of southern Taiwan for 4-5 months during the 2010 typhoon season to measure directional wave spectra, wind speed and direction, and momentum fluxes. Data were recorded for one severe tropical storm and three tropical cyclones, including Super Typhoon Megi. The data were valuable, but the maximum wind speed observed during the deployment was $26 \mathrm{~m} \mathrm{~s}^{-1}$. No matter what in situ instruments are deployed, it is unlikely they will get observations in the eye of the storm.

If the maximum wind speed, radius of maximum wind, and forward speed of two tropical cyclones in the open ocean were the same, the spatial variation of the wave fields they generated would presumably be the same. But it would be impossible to verify that with in situ instruments because of their very limited sampling of the area surrounding any particular storm. The solution to these sampling problems is a wave measurement system deployed on an aircraft that can document an extensive area of tropical cyclones.

\section{NOAA Wide Swath Radar Altimeter}

There was no peer-reviewed information available on the NOAA Wide Swath Radar Altimeter (WSRA) directional wave spectra when the Tamizi and Young (2020) paper appeared. The airborne WSRA is a $16-\mathrm{GHz}$ digital beamforming radar altimeter developed by ProSensing with funding from the NOAA Small Business Innovative Research (SBIR) and Joint Hurricane Testbed (JHT) programs, and additional support for the development of the microstrip array antenna from the Center for Advanced Sensor and Communication Antennas (CASCA) at the University of Massachusetts 
Amherst. The WSRA uses 80 narrow beams spread cross-track over $\pm 30^{\circ}$ from the nadir-oriented antenna to measure the backscattered power and range to the sea surface.

At approximately a $10-\mathrm{Hz}$ rate, the ranges from the 64 narrow beams nearest nadir $\left( \pm 23^{\circ}\right)$ are multiplied by the cosines of their various incidence angles to determine the vertical distance to each position across the swath. The aircraft altitude is determined by averaging the 64 heights on each cross-track raster line and then filtering the result along-track to eliminate wave contamination. Subtracting the individual narrow beam vertical distances from the aircraft altitude produces a topographic map of the waves as the aircraft advances.

The WSRA operates unattended on the aircraft and provides continuous real-time reporting of several data products: 1) significant wave height; 2) directional ocean wave spectra; 3 ) the wave height, wavelength, and direction of propagation of the primary and secondary wave fields; 4) rainfall rate; and 5) sea surface mean square slope (mss). Walsh et al. (2014) describes both the rainfall rate and the mss measurement capabilities.

During hurricane flights the data products are transmitted in real time from the NOAA WP-3D aircraft through a satellite data link to a ground station and on to the National Hurricane Center (NHC) for use by the forecasters for intensity projections and incorporation in hurricane wave models. PopStefanija et al. (2021) describe the WSRA hardware, data acquisition, and processing in detail.

The NOAA WSRA had two predecessors that no longer exist, the NASA Surface Contour Radar (Walsh et al. 1989) and the NASA Scanning Radar Altimeter (Wright et al. 2001). The WSRA is now the only instrument that can provide highdensity measurements of the complex wave topography and directional wave spectra over a large area surrounding the eye of the storm and do it in real time.

\section{Targeted high-density hurricane measurements}

NOAA WP-3D aircraft N43RF carried the WSRA into Hurricane Lorenzo on 29 September 2019 and that flight will be used to demonstrate the potential of the WSRA to document the detailed variation of the wave field in the vicinity of hurricanes. The flight occurred in open water in the central North Atlantic basin when Lorenzo was about halfway between Florida and Africa where no in situ wave measurements were available. Figure 1 shows the track of Lorenzo and the locations of the 135 WSRA directional wave spectra. Individual spectra will be referred to by their sequence number, which can easily be identified in Fig. 1.

Figure 2 shows the National Hurricane Center (NHC) advisory archive estimates of maximum surface wind speed for Lorenzo. The circles indicate the maximum surface wind speed observed by the Stepped Frequency Microwave Radiometer (SFMR) (Uhlhorn and Black 2003; Klotz and Uhlhorn 2014) on the NOAA aircraft carrying the WSRA for the flight on 29 September 2019 and the previous day. The NHC Advisory Archive and the SFMR agree quite well on 28 September but the SFMR is significantly lower on 29 September.

A second NOAA aircraft (N42RF) flew approximately the same flight pattern as the aircraft carrying the WSRA at about

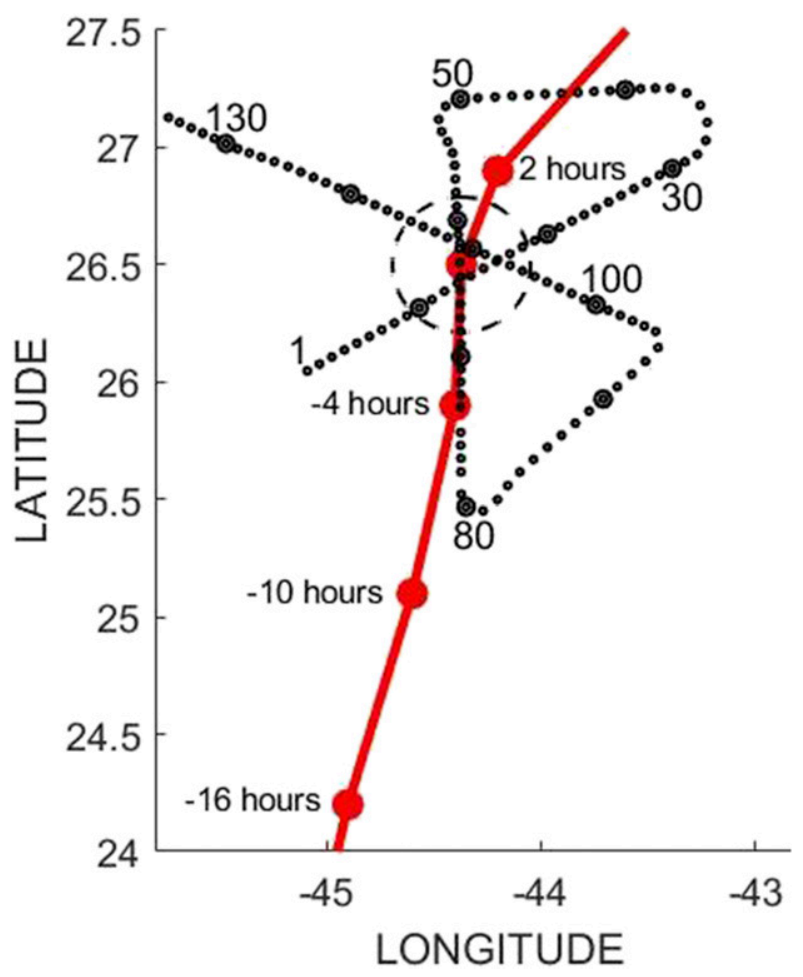

FIG. 1. Dots indicate the locations of 135 directional wave spectra produced by the WSRA during the flight into Hurricane Lorenzo on 29 Sep 2019. The dot at every tenth location is enlarged, and spectrum locations 1, 30, 50, 80, 100, and 130 are identified. The red curve is the track of Lorenzo, and four red dots indicate the National Hurricane Center (NHC) advisory archive locations of Lorenzo at the approximate times indicated relative to the second $\left(26.5^{\circ} \mathrm{N}, 44.37^{\circ} \mathrm{W}\right.$ at $\left.1908 \mathrm{UTC}\right)$ of the three eye penetrations made by the NOAA aircraft carrying the WSRA. The relative times $(-16,-10,-4,2 \mathrm{~h})$ were at $0300,0900,1500$, and 2100 UTC 29 Sep 2019. The dashed circle is a 32-km estimate for the radius of maximum wind.

the same time on 29 September, but at $2.4 \mathrm{~km}$ instead of the $3-\mathrm{km}$ altitude of N43RF. The second aircraft SFMR indicated a maximum surface wind speed of $40 \mathrm{~m} \mathrm{~s}^{-1}$. The ocean surface winds measured by SFMR during the flight showed that Lorenzo had weakened more than projected in the NHC Advisory Archive.

In a postseason report, NHC indicated that Lorenzo had reached its peak category 5 intensity of $140 \mathrm{kt}\left(1 \mathrm{kt} \approx 0.51 \mathrm{~m} \mathrm{~s}^{-1}\right)$ around 0300 UTC 29 September and then weakened even faster than it had strengthened, becoming a category 2 storm by 1800 UTC, just $15 \mathrm{~h}$ after its peak (https://www.nhc.noaa.gov/ data/tcr/AL132019_Lorenzo.pdf).

The top panel of Fig. 3 is a storm-relative plot of the $3-\mathrm{km}$ aircraft altitude downwind vector at the spectra locations. The reader can assess how reasonable our $32-\mathrm{km}$ estimate for the radius of maximum wind (RMW) was. The storm-relative plot of the wave height and propagation directions of the primary and secondary wave fields in the bottom panel of Fig. 3 shows that the largest waves were in the right forward quadrant. 


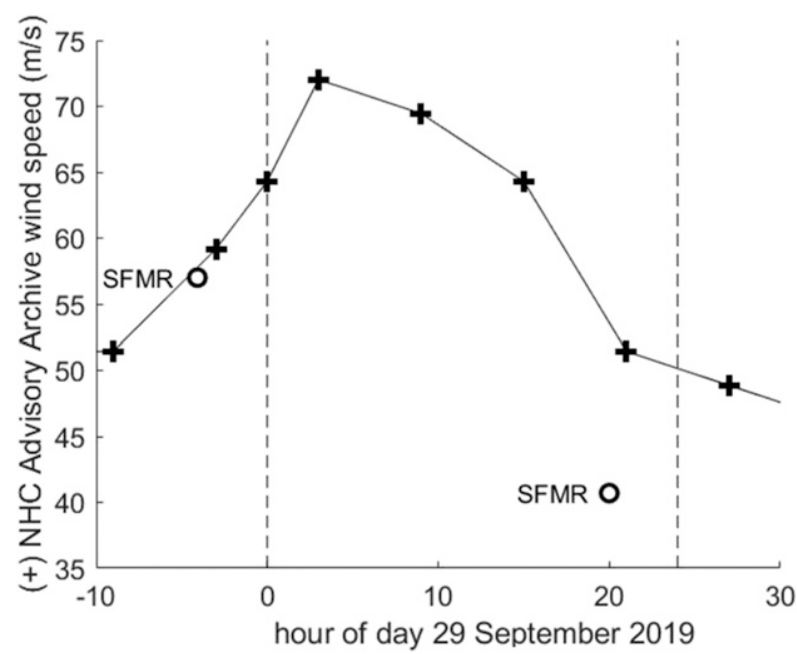

FIG. 2. The + symbols indicate the NHC advisory archive estimates of maximum surface wind speed. The circles indicate the maximum surface wind speed observed by the SFMR on the NOAA aircraft carrying the WSRA.

The maximum wave height observed was $9.3 \mathrm{~m}$, which is reasonable for the weak category 2 hurricane that Lorenzo appeared to be at that time.

Rapid changes in aircraft altitude or roll attitude or hardware problems can degrade the wave spectra. The output data placed in the archive postflight are not hand edited to eliminate poor quality spectra. There are generally only a few problem spectra. In the bottom panel of Fig. 3, the radials associated with $3 \%$ of the spectra (numbers $18,44,45$, and 104) were deleted. Two of them (18 and 104) were near the eyewall.

The Fig. 3 hurricane flight pattern is typical with radial flight lines spaced at $60^{\circ}$ azimuths. There was a long transit to reach Lorenzo so the legs were necessarily shortened. With a shorter transit time, the flight legs typically extend closer to $200 \mathrm{~km}$ from the eye or there are additional eye passes to increase the azimuthal resolution.

The top panel of Fig. 4 is a grayscale coded image of 600 cross-track raster lines of WSRA wave topography as the aircraft flew northeast at spectral location 2. There are two wave systems apparent in the image. Long waves propagated across the swath while short waves propagated along the flight track.

We will refer to the directional wave spectrum generated by the two-dimensional FFT applied to the wave topography as the encounter spectrum because it documents the wavelengths and propagation directions of the waves as the aircraft encountered them. In the time it takes to acquire the wave topography data, waves propagating in the aircraft flight direction will move away from the aircraft and appear to have a longer wavelength while waves propagating in the opposite direction will appear shorter in the wave topography. Waves propagating in other directions will generally appear to have both different wavelengths and directions of propagation. Since the speed and flight direction of the aircraft are known, it is trivial to Dopplercorrect the encounter spectrum, transforming it into the actual directional wave spectrum.

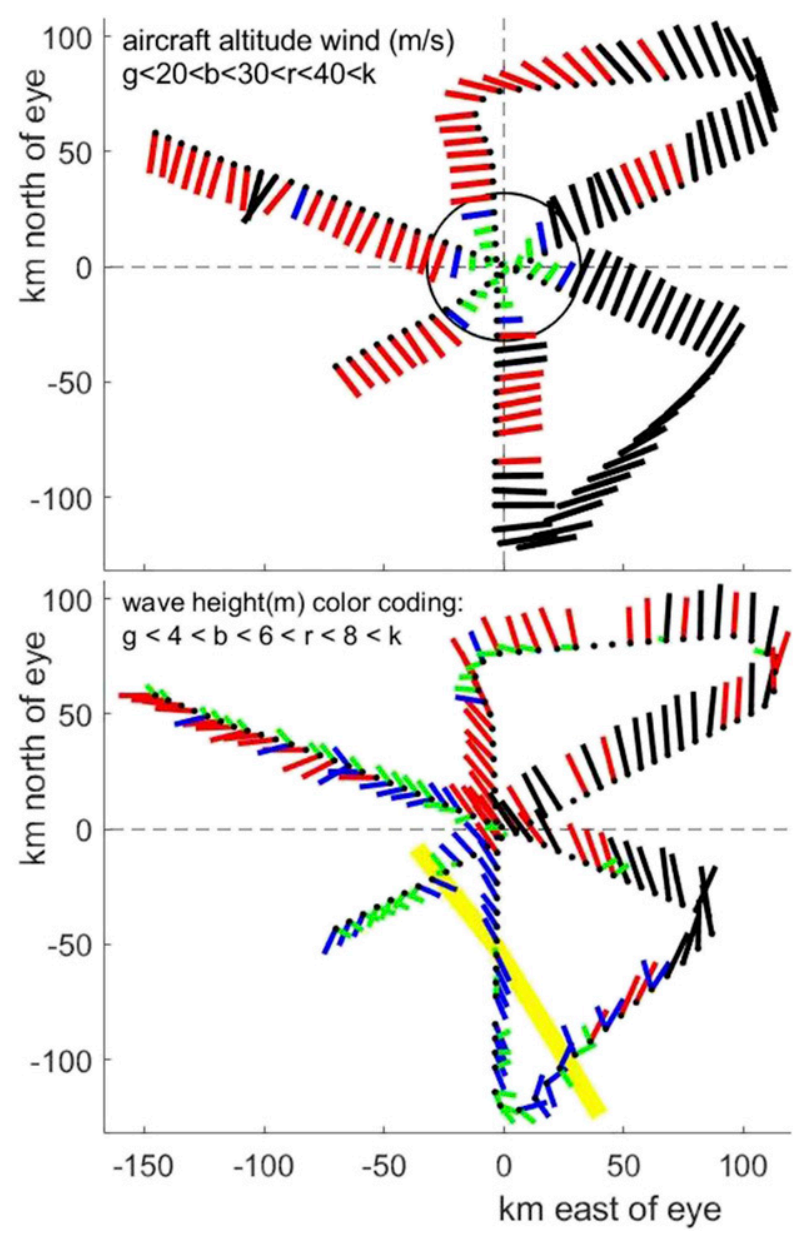

FIG. 3. Black dots indicate the storm-relative locations of the 135 spectra of Fig. 1 with color-coded (green, blue, red, black) (top) downwind radial lengths proportional to the aircraft 3-km altitude wind speed and (bottom) radials proportional to wave height extending in the propagation directions of the primary and secondary (if identified) wave systems. The circle of $32-\mathrm{km}$ radius is an estimate of the radius of maximum wind.

It is not possible to tell the direction of propagation of waves from a single snapshot of the topography. The two-dimensional FFT that generates the encounter spectrum deposits half the energy of each wave system in a real lobe corresponding to the wave propagation direction and half in an identical artifact lobe propagating in the opposite direction. Deleting the artifact lobes will be discussed later.

The bottom panel of Fig. 4 shows the resulting directional wave spectrum after the artifact lobes have been deleted and the real lobes have been Doppler-corrected for wave motion during data acquisition. The WSRA output directional wave spectra are truncated to $65 \times 65$ point arrays centered on zero wavenumber with $0.00245 \mathrm{rad} \mathrm{m}^{-1}$ resolution spanning $\pm 0.08 \mathrm{rad} \mathrm{m}^{-1}( \pm 80 \mathrm{~m})$ in $k$ space. To better show the energetic portion of the spectrum, the Fig. 4 spectrum has been enlarged, bounded by the $100-\mathrm{m}$ wavelength circle. All the spectra in this paper are plotted with nine contours linearly spaced from $10 \%$ to $90 \%$ of the spectral peak. 

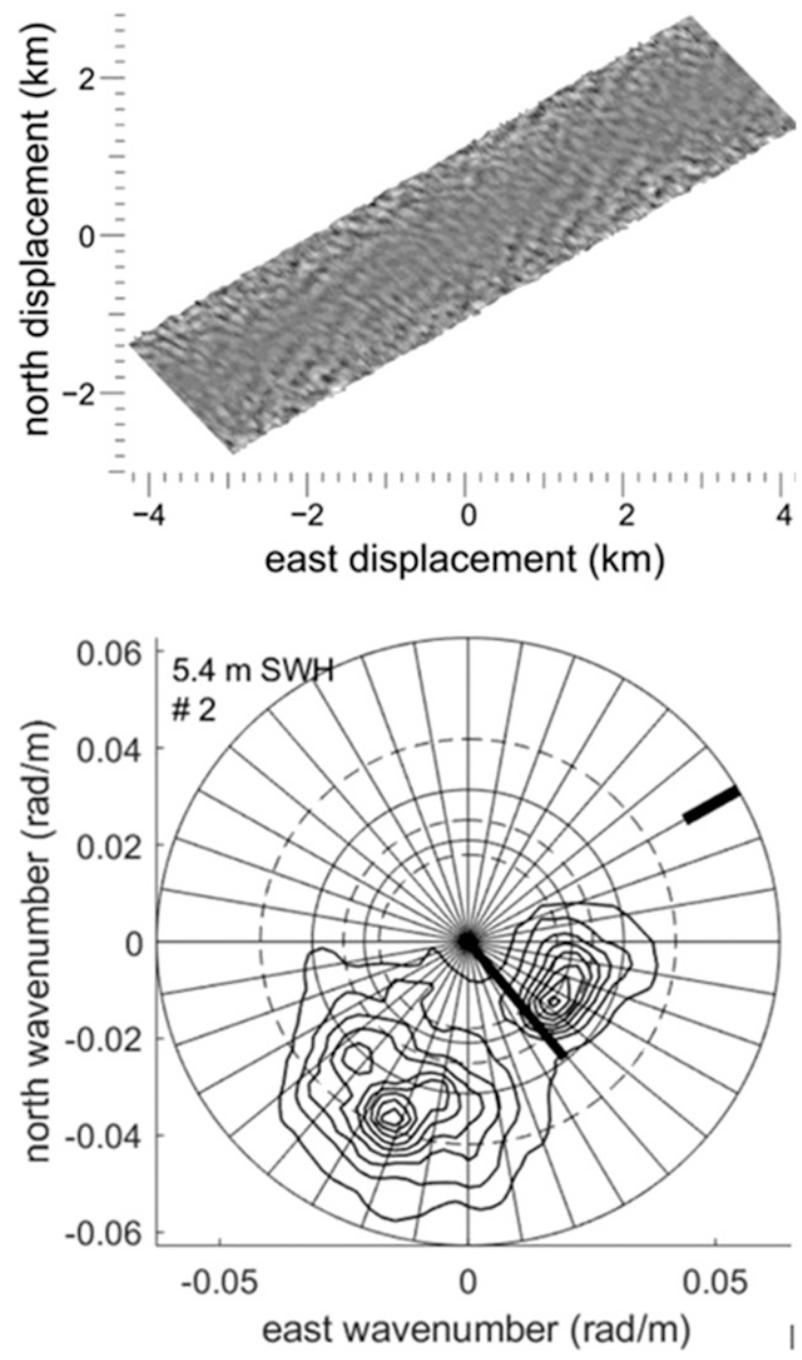

FIG. 4. (top) WSRA grayscale coded wave topography at spectral location 2. (bottom) Directional wave spectrum at location 2 with a downwind radial of length equal to 0.001 times wind speed at the aircraft altitude. Nine contours are linearly spaced at $0.1-0.9$ of the peak spectral variance. The thick black line at the $100-\mathrm{m}$ wavelength circle indicates the aircraft flight direction.

The automated processing zeroes out an area surrounding the spectral peak and the maximum value of what remains is determined. If a saddle point exists between the spectral peak and the secondary peak, the azimuth of its minimum value is taken as the partition boundary between the primary and secondary wave fields (PopStefanija et al. 2021). The automated processing does not try to identify a tertiary wave system.

Having the primary and secondary wave system characteristics automatically extracted and immediately available to plot as in Fig. 3 is convenient for assessing complex wave situations. For example, Lorenzo had been translating a little east of north. Figure 3 shows that the waves in the right front quadrant were nearly aligned with the local wind and were the largest in the hurricane due to the partial resonance with the storm forward motion.
Figure 5 shows three wave spectra $(28,81,126)$ that had quite different characteristics even though they were similar distances from the eye $(95,122,103 \mathrm{~km})$ and had about the same local wind speed at the aircraft altitude $\left(46,49,48 \mathrm{~m} \mathrm{~s}^{-1}\right)$. The top panel shows spectrum 28 from the right forward quadrant. It had the longest wavelength $(320 \mathrm{~m})$ and highest wave height of the three. That spectrum was the most narrow and nearly aligned with the local wind. Spectrum 81 , directly south of the eye, was also centered on the local wind direction but was very broad. West of the eye, spectrum 126 was propagating $50^{\circ}$ off the local wind direction but with an extrusion toward it.

\section{Artifact spectral lobe deletion}

The automated processing uses a hurricane wind model (Willoughby et al. 2006; Zhang and Uhlhorn 2012) to generate a wind forcing pattern to predict the propagation direction for waves of various lengths. In 10-km steps outward from the WSRA observation point, the square of the model wind component directed toward the observation point is integrated on every $5^{\circ}$ azimuth to generate an effective wind forcing pattern. For each $10-\mathrm{km}$ step outward from the observation point, an earlier position of the hurricane is used, determined by the transit time to the observation point at the group velocity of the ocean wavelength under consideration.

The wind forcing computation does not consider how the presence of land might affect the wave spectra either by blocking waves or limiting the wave growth fetch. The centroid of the wind forcing pattern is used to predict the propagation direction for waves of that wavelength.

Wind forcing patterns are computed for eight different wavelengths: $91,102,116,135,160,197,256$, and $366 \mathrm{~m}$. The distances from the observation point that the wind is integrated over vary from $129 \mathrm{~km}(6 \mathrm{~h})$ for the shortest wavelength to $516 \mathrm{~km}(12 \mathrm{~h})$ for the longest.

To eliminate the artifact lobes, the peak of the perfectly symmetrical encounter spectrum is determined. The two peak locations (real and artifact) are Doppler-corrected for wave motion during data acquisition, which will generally change both their wavelengths and propagation directions. The peak whose corrected propagation direction is closest to the wavelength-interpolated direction of the eight wavelength wind-weighting centroid directions is deemed the real lobe. Details of this iterative procedure are described by PopStefanija et al. (2021).

Computing earlier hurricane positions and the resulting wind field by assuming a constant wavelength and group velocity over hundreds of kilometers for each of the eight wavelengths arriving at the observation location is expedient. But since those waves and their group velocities would have evolved over time and distance, the earlier hurricane positions computed would not be optimum; when the radius of maximum wind is unknown, $40 \mathrm{~km}$ is used as a default. And the spatial variation of the actual wind field might be significantly different than the model wind field, and it might vary temporally. But the wind weighting prediction does not have to be 

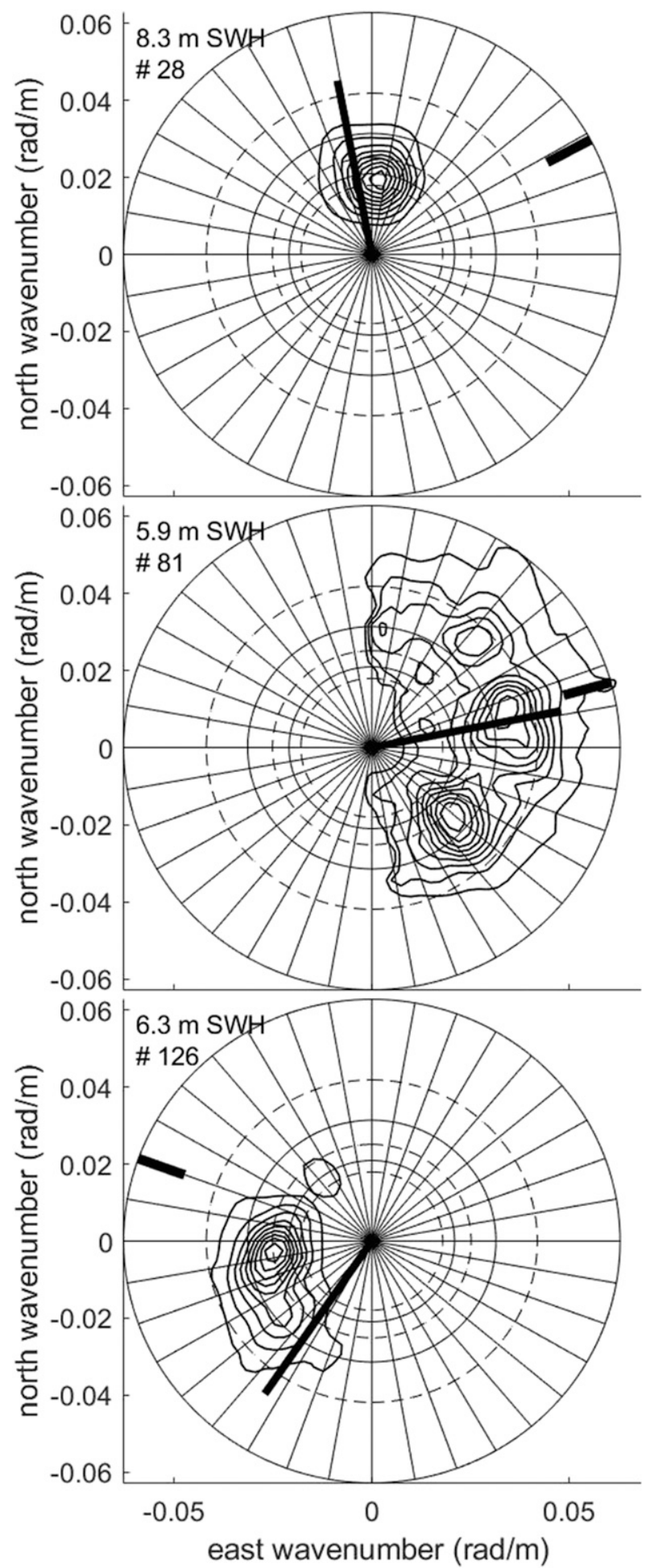

FIG. 5. Directional wave spectra at locations (top) 28 , (middle) 81, and (bottom) 126 with downwind radials of length equal to 0.001 times the wind speed at aircraft altitude and thick lines at the 100-m wavelength circle indicating the aircraft flight direction. Nine contours are linearly spaced at $0.1-0.9$ of the peak spectral variance. perfect. It only needs to be a little closer to the real propagation direction than the artifact lobe direction.

To protect against the possibility that an artifact spectral lobe might have been selected instead of the real lobe, there is also a standard array in the WSRA data product file in which all the encounter spectra were corrected as if everything was real and nothing was deleted. Then if investigators thought that a final output spectrum contained an artifact lobe, they could examine the same spectrum in the file where everything was retained and delete the alternate lobe without having to reprocess the data from the beginning.

\section{Wave spectra problem area}

There is one unusual situation that is problematical for determining wave spectra from wave topography. In the bottom panel of Fig. 3 between spectra 8 and $9(27 \mathrm{~km}$ west, $20 \mathrm{~km}$ south) the propagation direction of the dominant waves abruptly reversed. In that region there were waves propagating in both directions. When wave systems of comparable wavelength propagate in opposite directions the WSRA will select one and delete the other, hopefully choosing the more dominant as the two wave systems evolve spatially.

The automated artifact lobe deletion algorithm generally does an excellent job. The blue dots in Fig. 6 show how the computed wind-weighting centroid varied in the problematic region where waves were propagating in opposite directions. Spectra 7-10, enlarged to the 150-m circle for clarity, are shown with the artifact lobes retained. The aircraft altitude wind vectors in those spectra showed a rapid reduction in the strength of the wind $\left(35,33,24\right.$, and $\left.16 \mathrm{~m} \mathrm{~s}^{-1}\right)$ that was generating the southeast waves as the aircraft entered the eye.

The southeast propagating waves were diminishing as the aircraft entered the eye and the northwest propagating waves were strengthening. As a result, the centroids of the wind weighting computation were positioned between the two directions. For spectra 7 and 8 the automated processing selected the southeast propagating waves as "real." At the location of spectrum 7 the wind weighting centroid direction was clearly closer to the southeast propagating waves. For spectrum 8 the wind weighting centroid direction shifted more westerly for the shorter wavelengths. But the longest wavelength was still closer to the southeast waves.

For spectrum 9 the 366-m wavelength centroid direction was still closer to the southeast waves, but the real-lobe selection process chose the northwest waves. It uses the Dopplercorrected wavelengths of the real and artifact spectral peaks to interpolate the eight wavelengths $(91,102,116,135,160,197$, 256 , and $366 \mathrm{~m}$ ) to obtain wind-weighting centroid azimuths. The northwest peak was closer to the interpolated windweighting azimuth. For spectrum 10 the wind-weighting azimuth was clearly closer to the northwest waves.

There were two other locations where there was a sudden reversal of wave propagation direction from northwest to southeast: between spectra 70 and 71 and between spectra 85 and 84 . The yellow line in the bottom panel of Fig. 3 appears to be a boundary between the dominance of the northwest and southeast waves. 

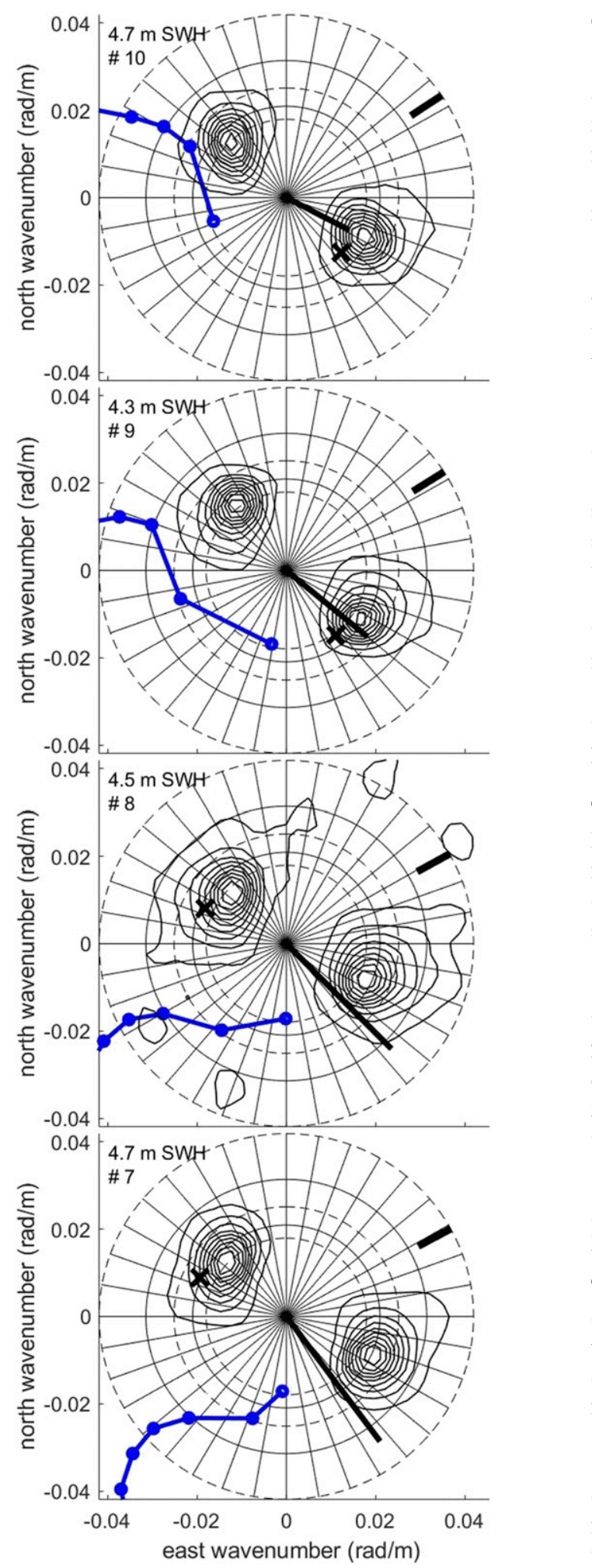

FIG. 6. Directional wave spectra 7-10 with the artifact lobes retained. The blue dots indicate the azimuth of the wind-weighting centroid for some of the eight wavelengths for which it was computed. The X mark shows the $180^{\circ}$ symmetrical position from the spectral peak of the "real" lobe selected by the automated processing and identifies the "artifact" lobe.

\section{In the eye of the storm}

The first and third eye penetrations were separated by $82 \mathrm{~min}$ and Hurricane Lorenzo traveled $21 \mathrm{~km}$ in that time. But if the characteristics of the storm did not change, the stormrelative wave field could be assumed unchanged.

Figure 7 shows a grayscale coded image of 600 cross-track raster lines of WSRA wave topography centered on the eye and associated with spectrum 63 . Figure 8 shows wave spectra 13,63 , and 110 with the artifact lobes retained. Those spectra were nearest the center for the three eye passes. For clarity the spectra have been enlarged, bounded by the $150-\mathrm{m}$ wavelength circle.

The Doppler correction applied to an encounter spectrum assumes all spectral components are real. In general, the first order Doppler correction shifts all wave components, real and artifact, in the aircraft flight direction. The real lobes are shifted into their proper positions and the artifact lobes are shifted in the wrong direction, out of their symmetrical positions. As a result, Doppler correction can identify the real and artifact spectral lobes without a priori information whenever there are wave spectra from significantly different flight directions in the same area.

Figure 8 demonstrates that the wave field at the center of the eye was unimodal and propagating toward the northwest. The propagation directions at the spectral peaks were $322.4^{\circ}$ (spectrum 13 ), $326.1^{\circ}$ (63), and $326.1^{\circ}$ (110), nearly identical. The X mark in Fig. 8 , indicating the $180^{\circ}$ perfectly symmetrical locations of the northwest spectral peaks, shows that the artifact lobes in the three spectra are badly mismatched with each other in both wavelength and propagation direction and each is shifted out of its symmetrical position with the real lobe in the aircraft flight direction.

The dominant wavelength in the eye showed a decreasing trend over the three passes [354 m (spectrum 13), $299 \mathrm{~m}$ (63), $278 \mathrm{~m}$ (110)]. Averaging wavelengths from the three spectra closest to the eye on each pass resulted in the same trend (345, 309 , and $298 \mathrm{~m}$ ). The wave height was trending higher over the three passes, whether considering the spectra closest to the eye $(6.3,6.7,7.2 \mathrm{~m})$ or three-spectra averages $(6.3,6.9,7.6 \mathrm{~m})$.

The trends may have been temporal, or spatial because Lorenzo's track was shifting during the time of the flight, or because the flight tracks did not all reach the center of the eye. The minimum wind speeds at the aircraft altitude for the three eye passes were $8.3,0.6$, and $3.4 \mathrm{~m} \mathrm{~s}^{-1}$. An analysis of the $1-\mathrm{Hz}$ wind vectors at the aircraft altitude indicated the center of the eye was at $26.46^{\circ} \mathrm{N}, 44.45^{\circ} \mathrm{W}$ during the first pass, about $7 \mathrm{~km}$ from the track, and at $26.61^{\circ} \mathrm{N}, 44.32^{\circ} \mathrm{W}$ during the third pass, about $4 \mathrm{~km}$ from the track.

But independent of the cause, the spectra show the level of detail on the wave field provided by the WSRA. The average forward speed of Lorenzo over the $16 \mathrm{~h}$ between $0300 \mathrm{UTC}$ and the second eye penetration at 1908 UTC was $4.5 \mathrm{~m} \mathrm{~s}^{-1}$. If a numerical wave model predicted that there would be $300 \mathrm{~m}$ waves of $7-\mathrm{m}$ height propagating toward $325^{\circ}$ in the eye of Lorenzo, it would be impressive since the $10.8 \mathrm{~m} \mathrm{~s}^{-1}$ group velocity for $300-\mathrm{m}$ waves is 2.4 times faster than Lorenzo's forward speed. How did those long waves end up in the eye 


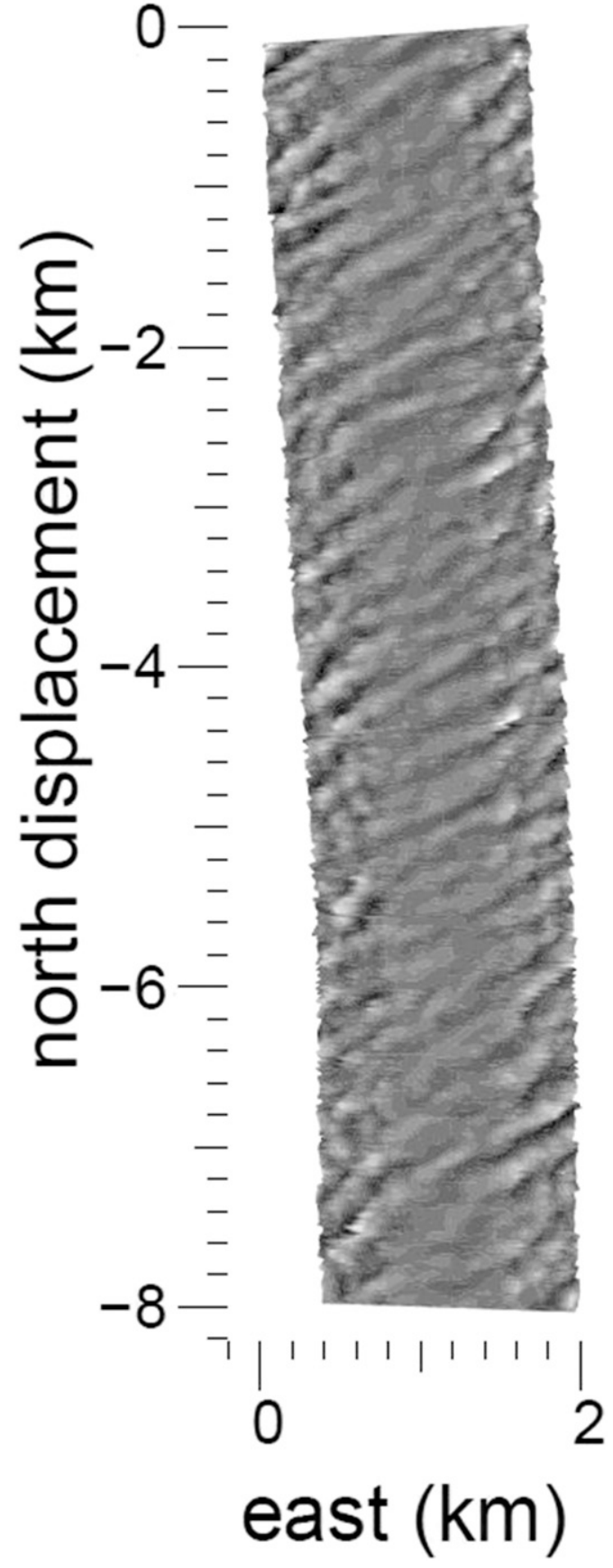

FIG. 7. WSRA grayscale coded wave topography at spectral location 63 .

when they were traveling much faster than the storm that created them?

The bewilderment expressed in the previous sentence was not to suggest that the observed waves in the eye could not be explained. It was just intended to indicate that wave generation in a hurricane is a complex process. Figure 3 shows northwest propagating waves in the eye without any indication of a northwest wind field in the right rear quadrant of Lorenzo.

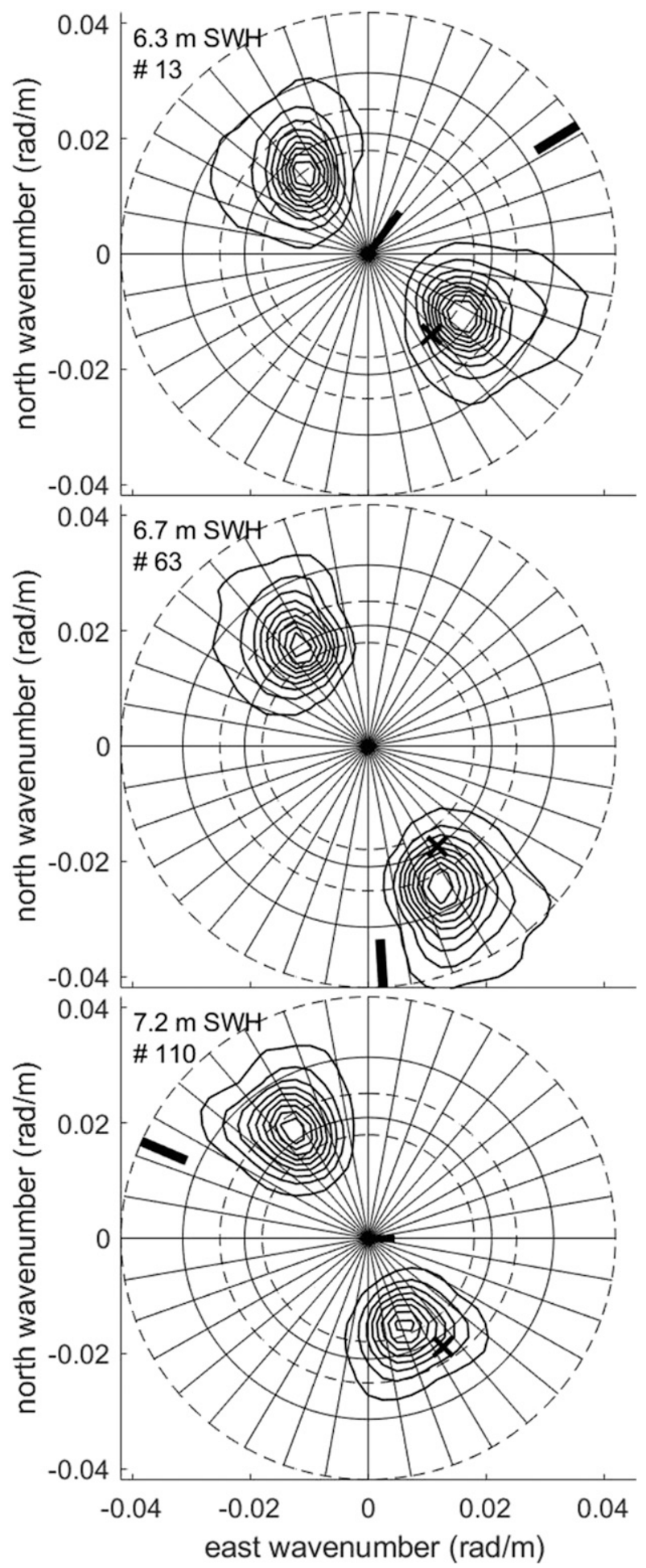

FIG. 8. Directional wave spectra at locations 13,63 and 110 with the $\mathrm{X}$ indicating the $180^{\circ}$ symmetrical positions relative to the real lobe spectral peaks. Radial lengths are equal to 0.001 times downwind speed at aircraft altitude. The thick black line at the $150-\mathrm{m}$ circle indicates aircraft flight direction. Nine contours spaced at $0.1-0.9$ of peak spectral variance. 


\section{Summary}

Tamizi and Young (2020) and Collins et al. (2018) demonstrated the need for an airborne system that can provide comprehensive measurements of the directional wave spectrum of individual tropical cyclones within the eye of the storm and for hundreds of kilometers surrounding it. The WSRA can provide that ocean wave data. Since NOAA aircraft frequently fly into a hurricane every $12 \mathrm{~h}$, the environmental data collected by their operational instruments (https:// www.aoml.noaa.gov/wp-content/uploads/2020/05/3_2020HFP_ InstrumentDescriptions.pdf) can well document the evolving characteristics of individual tropical cyclones and provide a basis for verifying and improving the performance of numerical wave models.

If a numerical wave model could accurately produce the details of the WSRA directional wave spectrum spatial variation throughout the vicinity of Lorenzo, as well as a dozen other tropical cyclones of different sizes, strengths, and forward speeds, you could have confidence in its predictions. Data from 15 WSRA flights into six tropical cyclones are available now at https://www.prosensing.com/wsra-level-4-data/ and more will be added soon.

In addition to hurricane activity, the WSRA has supported the 2020 Atlantic Tradewind Ocean-Atmosphere Mesoscale Interaction Campaign (ATOMIC) out of Barbados and the CalWater 2015 experiment off the coast of California. The ATOMIC data are available at the ProSensing website and CalWater data will be added.

Acknowledgments. The NOAA Aircraft Operations Center installs and operates the WSRA. ProSensing activity related to the WSRA was supported by Contract 1305L319PNRMJ0171 from the NOAA/Physical Sciences Laboratory. We greatly appreciate the insightful comments of the reviewers.

Data availability statement. At https://www.prosensing.com/ wsra-level-4-data/ there is a README file describing the variables in the WSRA netCDF output files. WSRA netCDF files containing output data are identified by the year, month, and day the observations began. For example, WSRA-L4-20190929I1.nc is the file containing the data from the Hurricane Lorenzo flight on 29 September 2019. All data are available to the public without limitation.

\section{REFERENCES}

Collins, C. O., H. Potter, B. Lund, H. Tamura, and H. C. Graber, 2018: Directional wave spectra observed during intense tropical cyclones. J. Geophys. Res. Oceans, 123, 773-793, https:// doi.org/10.1002/2017JC012943.

Klotz, B. W., and E. W. Uhlhorn, 2014: Improved stepped frequency microwave radiometer tropical cyclone surface winds in heavy precipitation. J. Atmos. Oceanic Technol., 31, 23922408, https://doi.org/10.1175/JTECH-D-14-00028.1.

PopStefanija, I., C. W. Fairall, and E. J. Walsh, 2021: Mapping of directional ocean wave spectra in hurricanes and other environments. IEEE Trans. Geosci. Remote Sens., https://doi.org/ 10.1109/TGRS.2020.3042904, in press.

Tamizi, A., and I. R. Young, 2020: The spatial distribution of ocean waves in tropical cyclones. J. Phys. Oceanogr., 50, 2123-2139, https://doi.org/10.1175/JPO-D-20-0020.1.

Uhlhorn, E. W., and P. G. Black, 2003: Verification of remotely sensed sea surface winds in hurricanes. J. Atmos. Oceanic Technol., 20, 99-116, https://doi.org/10.1175/1520-0426(2003) $020<0099$ :VORSSS $>2.0$.CO;2.

Walsh, E. J., D. W. Hancock III, D. E. Hines, R. N. Swift, and J. F. Scott, 1989: An observation of the directional wave spectrum evolution from shoreline to fully developed. J. Phys. Oceanogr., 19, 670-690, https://doi.org/10.1175/1520-0485(1989) 019<0670:AOOTDW > 2.0.CO;2.

—, I. PopStefanija, S. Y. Matrosov, J. Zhang, E. Uhlhorn, and B. Klotz, 2014: Airborne rain-rate measurement with a wide-swath radar altimeter. J. Atmos. Oceanic Technol., 31, 860-875, https://doi.org/10.1175/JTECH-D-13-00111.1.

Willoughby, H. E., R. W. R. Darling, and M. E. Rahn, 2006: Parametric representation of the primary hurricane vortex. Part II: A new family of sectionally continuous profiles. Mon. Wea. Rev., 134, 1102-1120, https://doi.org/10.1175/ MWR3106.1.

Wright, C. W., and Coauthors, 2001: Hurricane directional wave spectrum spatial variation in the open ocean. J. Phys. Oceanogr., 31, 2472-2488, https://doi.org/10.1175/1520-0485(2001)031<2472: HDWSSV $>2.0 . \mathrm{CO} ; 2$.

Zhang, J. A., and E. W. Uhlhorn, 2012: Hurricane sea surface inflow angle and an observation-based parametric model. Mon. Wea. Rev., 140, 3587-3605, https://doi.org/10.1175/MWR-D11-00339.1. 